\title{
MIBG detection of hepatic neuroblastoma: correlation with CT, US and surgical findings
}

\author{
D. A. Dessner, M. A.DiPietro, B.L.Shulkin
}

Departments of Radiology (Section of Pediatric Radiology) and Internal Medicine (Division of Nuclear Medicine), University of Michigan Hospitals, Ann Arbor, Michigan, USA

\begin{abstract}
Metaiodobenzylguanidine (MIBG) imaging is used in the diagnosis, staging and follow-up of virtually every case of neuroblastoma seen at our institution. Normal sites of MIBG uptake include the liver and therefore difficulties have been predicted and encountered in the diagnosis of hepatic neuroblastoma due to inability to separate abnormally increased tracer deposition from normal hepatic activity. We reviewed every MIBG ( $\mathrm{I}^{123}$ and $\mathrm{I}^{131}$ ) study performed at our pediatric hospital over a 4 year period encompassing 88 patients, 67 of whom had biopsy proven neuroblastoma. Hepatic findings onMIBG studies were compared with concurrent abdominal CT and US studies in all 67 patients. The clinical records of all patients with abnormal MIBG scans or abnormal CT or US studies of the liver were also reviewed. Eight patients were found to have abnormal liver findings on one or more imaging studies (MIBG, CT, or US). There were 3 true positive MIBG studies, one of which was an early study in a patient who later went on to have one of the false positive studies. Two patients had false positive MIBG scans for liver neuroblastoma. MIBG failed to detect liver involvement in 4 patients.
\end{abstract}

Metaiodobenzylguanidine (MIBG) imaging is widely used in the diagnosis and staging of neuroblastoma [1]. Its high sensitivity and specificity have resulted in its use in diagnosis and follow-up in virtually all cases of neuroblastoma seen at our institution [2].

Normal sites of uptake of MIBG include liver which can make the detection of disease involving the liver problematic [3]. We undertook this retrospective review to determine the utility of MIBG scintigraphy for the detection of hepatic involvement in neuroblastoma, confirmed surgically or by other imaging techniques or both.

Correspondence to: M. A. DiPietro, MD, Department of Radiology, C.S. Mott Children's Hospital, University of Michigan Hospitals, Ann Arbor, Michigan 48109-0252, USA
We also discuss whether incorrect assessment of hepatic involvement by MIBG liver scintigraphy resulted in incorrect or delayed diagnosis or staging and describe the clinical and anatomic settings in which potential pitfalls are most likely to be encountered.

\section{Materials and methods}

All MIBG studies $\left(\mathrm{I}^{123}\right.$ and $\left.\mathrm{I}^{131}\right)$ performed on the pediatric population at our children's hospital from January, 1988 to November, 1992 were reviewed. Eighty-eight children had one or more studies. Sixtyseven children had pathologically proven neuroblastoma. All abdominal computed tomography (CT) and ultrasound (US) interpretations on those 67 patients were reviewed noting the presence of abnormal findings in the liver. Hepatic findings on MIBG and concurrent CT and US studies were compared. Clinical information and medical records were teviewed on all patients with abnormal MIBG or abnormal CT or US studies of the liver.

\section{Results}

Eight patients had abnormal liver findings on one or more of these studies (MIBG, CT, US). MIBG hepatic uptake in the liver was abnormal in 5 patients. Of these, 3 were confirmed cases of hepatic involvement by neuroblastoma. CT detected 5 of 6 cases of hepatic neuroblastoma as well as abnormalities not due to neuroblastoma. Ultrasound was used in 2 patients, both of whom had hepatic neuroblastoma shown at sonography, including one case where MIBG and CT were not interpreted as abnormal. Fiftynine of the 67 patients ( $88 \%$ ) had no evidence of liver involvement with neuroblastoma using any modality (MIBG, CT or ultrasound). Liver biopsies were not routinely performed and for the purposes of this study, these patients were considered to have true negative MIBG studies of the liver.

Three patients had true positive MIBG studies, two with stage IV-S disease (Fig. 1) and one with stage IV disease where several focal liver metastases were detected with $\mathrm{I}^{131}$ MIBG as well as with CT. 

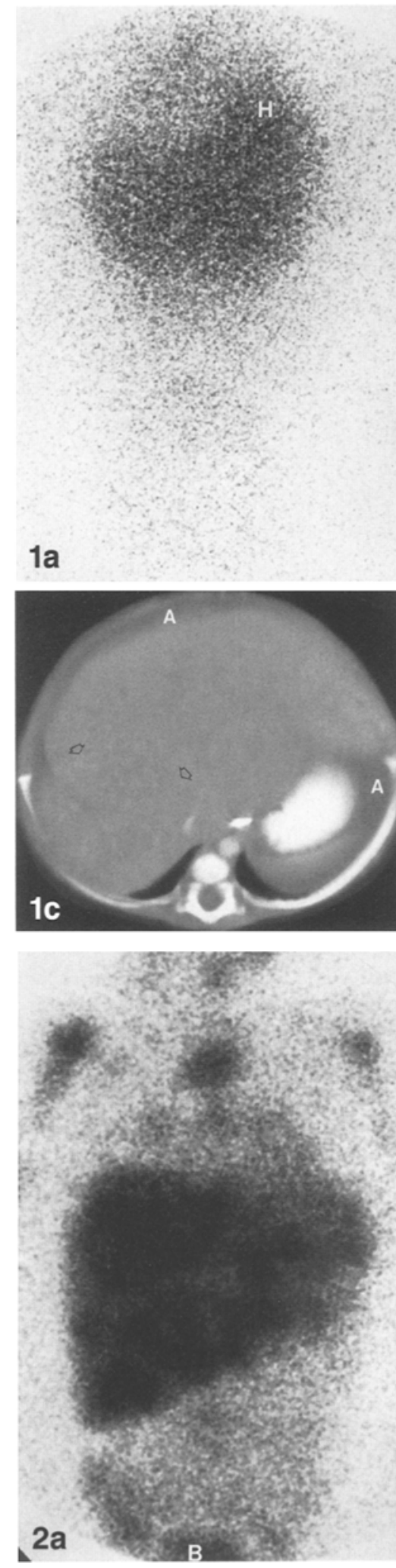
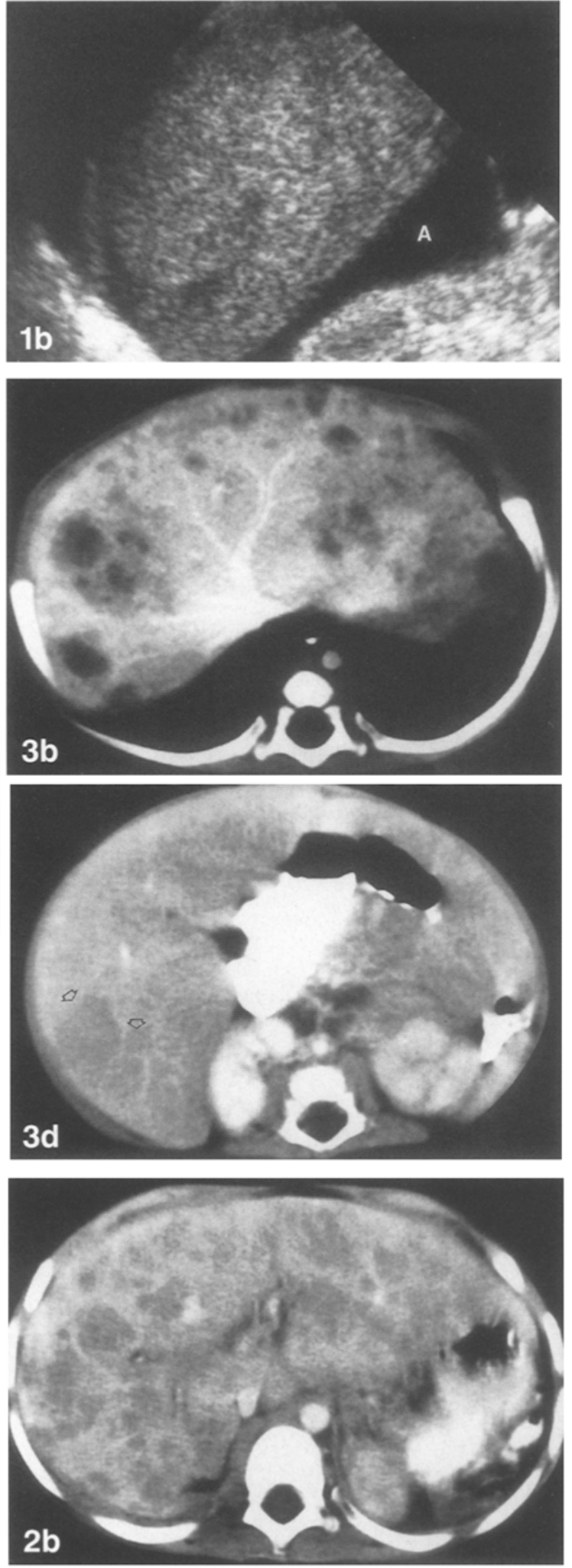

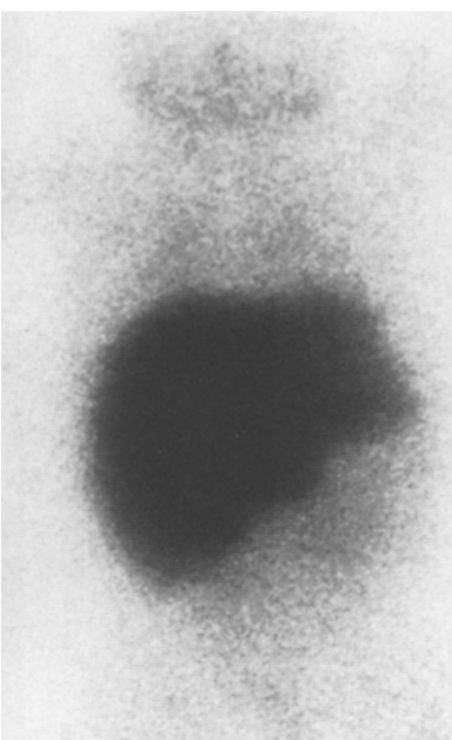

$3 a$

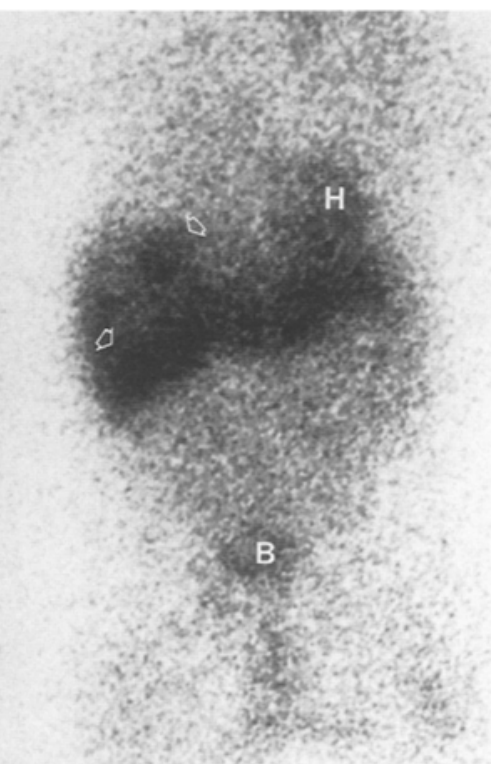

3c

Fig. 1a-c. 2-month-old male with hepatomegaly diagnosed with stage IV-S disease.

a ${ }^{131}$ MIBG scan shows enlarged liver suggesting metastatic disease. $H$, heart.

b US shows mottled, coarsened hepatic echotexture with ascites $(A)$. c CT shows inhomogeneous attenuation especially in the right lobe of an enlarged liver (arrows) with surrounding ascites $(A)$
Fig. 2a,b. Patient status post splenectomy for candida abscesses with fungal sepsis and fungal sinusitis. a ${ }^{123}$ MIBG scan shows an enlarged liver with mottled uptake suspicious for hepatic metastases. Note abnormal marrow uptake in humeri, right ilium, and uptake in the mediastinum. Note also the improved image clarity, definition and spatial resolution obtained with $\mathrm{I}^{123}$ as opposed to $\mathrm{I}^{131}$. $B$,bladder. b CT shows multiple low density lesions in the liver. Biopsy confirmed diagnosis of fungal abscesses
Fig. 3a-d. 6-week-old male initially presented with left adrenal mass and hepatomegaly. a $\mathrm{I}^{131}$ MIBG scan shows diffusely increased uptake in a markedly enlarged liver. b Concurrent CT shows multiple deposits of metastatic disease in the liver. $\mathbf{c}$ After treatment, $\mathrm{I}^{123}$ MIBG scan shows dramatic improvement in liver, but there are still foci of increased uptake in the inferior and superior right lobe of the liver (arrows). $H$, heart; $B$, bladder. d CT shows area of decreased attenuation in posterior segment of right lobe (arrows) 

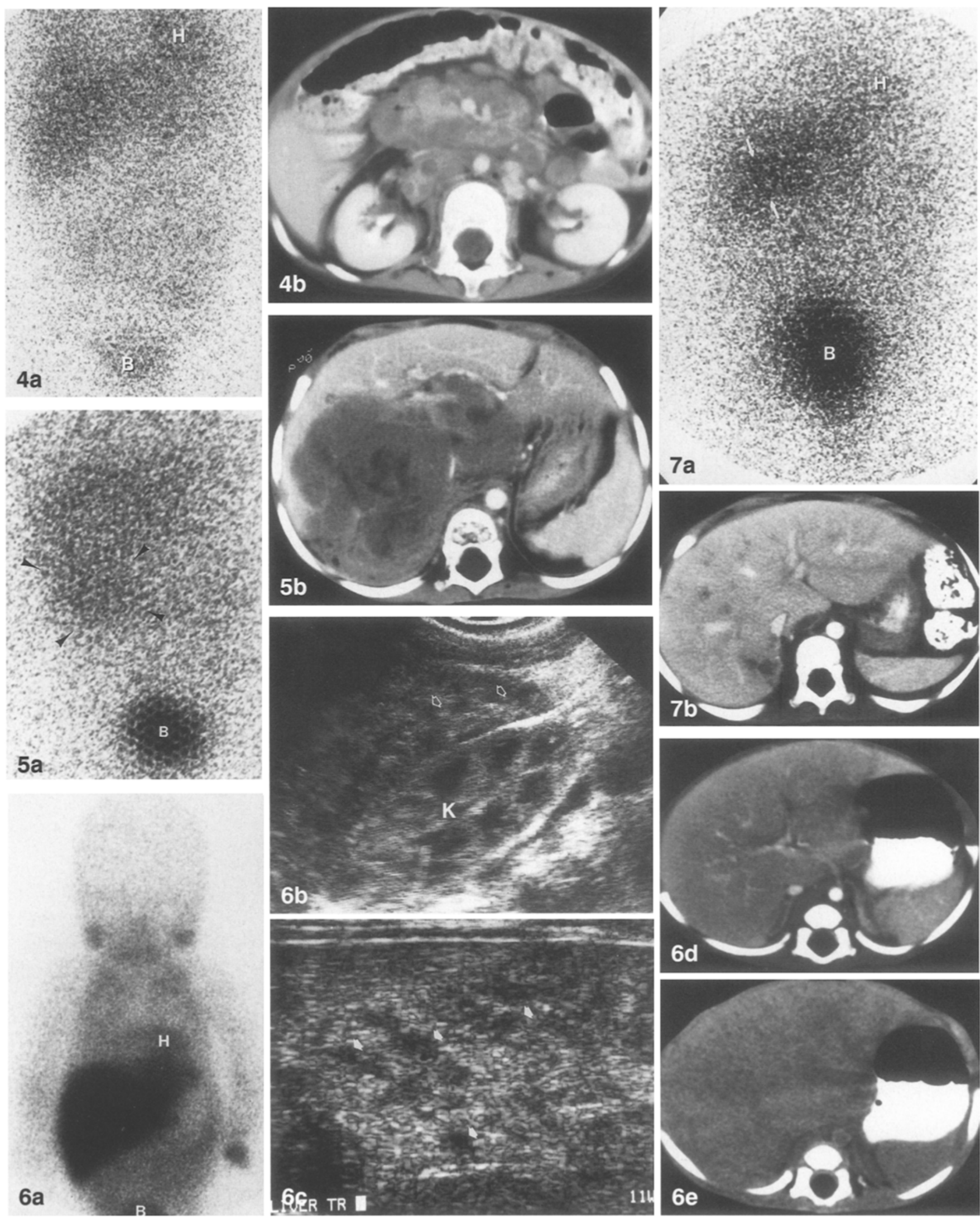
Two patients had false positive MIBG scans for neuroblastoma in the liver. One of these was a patient with proven diffuse and progressive hepatic candidiasis which resulted in a very mottled pattern of uptake in his enlarged liver (Fig.2). It could not be determined whether the abnormal image was due to multiple areas of increased uptake due to metastatic foci of neuroblastoma or whether there were actually multiple areas of decreased uptake where abscesses had formed, thereby distorting the normal homogeneous liver uptake. The other false positive MIBG study occurred in a patient with treated IV-S disease in whom an ${ }^{131}$ MIBG scan initially showed diffusely abnormal liver activity (one of the true positives in our series) (Fig. 3). However the patient eventually improved with only patchy areas of mildly increased uptake in the right lobe. The CT scan corroborated this observation, but open biopsy of the suspicious area revealed only ganglioneuroma with no malignant cells detected on numerous histologic sections.

MIBG studies failed to detect liver involvement by neuroblastoma in four patients. One false negative MIBG study was felt to be due to a change in the tumor's biological behavior which resulted in decreased uptake of the agent. In this patient, CT studies documented progressive disease, eventually leading to the patient's death, while serial $I^{131}$ MIBG studies showed gradual diminution in uptake, appearing normal three weeks before the patient died (Fig.4). A second false negative MIBG study occurred with recurrent disease which had directly invaded the undersurface of the liver. In this case, $I^{13}$ MIBG could not distinguish between hepatic invasion

Fig. 4a,b. 3-year-old female initially diagnosed with stage III disease status post chemotherapy, surgery and X-ray therapy. a I ${ }^{131}$ MIBG scan shows normal liver, heart $(H)$ and bladder $(B)$ activity and was read as normal. b CT shows bulky abdominal disease not detected by MIBG. Liver images (not shown) were suspicious for focal disease

Fig. 5a,b. Patient diagnosed at 18 months with abdominal mass and stage III disease. Status post chemotherapy, surgery and X-ray therapy with recurrent abdominal mass at 20 months of age. a I ${ }^{131}$ MIBG scan shows increased activity in right upper abdomen (arrows) but distinction between abdominal disease and liver invasion cannot be made. b CT shows tumor invading under surface of liver

Fig. 6a-e. 2-months-old male with hepatomegaly. a I ${ }^{123}$ MIBG scan shows normal uptake in salivary glands, heart $(H)$, and bladder $(B)$. The liver was felt to be within normal limits. b Sagittal US shows mottled hepatic echotexture. Two of the many diffuse hypoechoiclesions are labelled with arrows. $K$, kidney. c $7.5 \mathrm{MHz}$ linear array transducer shows the anterior $3 \mathrm{~cm}$ of the liver with multiple hypoechoic nodules between 1 and $3 \mathrm{~mm}$ in diameter. Four nodules are labelled. d Enhanced CT shows subtle inhomogeneity but was not read as abnormal. e Delayed CT images again suggest inhomogeneity but not unlike the appearance of the normal spleen and paraspinal muscles

Fig. 7a,b. Male patient presented with a right abdominal mass and stage IV disease. a $\mathbf{I}^{131}$ MIBG scan shows subtle increased uptake in right upper quadrant (arrows). $H$, heart; $B$, bladder. b CT shows multiple low attenuation lesions in liver, each less than $2 \mathrm{~cm}$. Low density in posterior right lobe represents superior aspect of adrenal primary tumor mass and recurrent abdominal bulky disease (Fig.5). A third false negative occurred with disseminated hepatic infiltration in a 2-month-old child with stage IV-S disease in whom there was no other tumor site. Uniformly increased $\mathrm{I}^{123}$ MIBG activity throughout the liver was not prospectively considered abnormal. Sonography showed a diffuse inhomogeneous echo texture suggesting infiltrating malignancy, which was proven at laparotomy. CT scan done at the time of presentation was likewise not prospectively interpreted as definitely abnormal although retrospectively, when compared to a study done six months later when the patient was in complete remission (achieved without therapy), the liver had been subtly inhomogeneous with some distortion of the normal hepatic vascular architecture (Fig. 6). The fourth false negative case was an $I^{131}$ MIBG study which failed to detect several small focal deposits in the liver (each under $2 \mathrm{~cm}$ in diameter) despite showing the primary lesion in the adrenal fossa (Fig. 7).

\section{Discussion}

MIBG is an excellent imaging agent for detection, staging, and monitoring of neuroblastoma $[1,4]$. However, false negative MIBG studies of the liver do occur and are usually due to the difficulty of detecting abnormally increased uptake against the normal hepatic background activity. This can make detection of diffuse disease seen in stage IV-S patients problematic, although 2 of 3 patients with IV-S disease did have prospective diagnoses of liver involvement made on MIBG scans. Detecting small lesions, even when focal, can be difficult and was a cause of one false negative MIBG study. This limitation in spatial resolution also resulted in the inability to detect hepatic invasion by recurrent primary disease, although concurrent CT scanning clearly showed the anatomic detail necessary to make the correct diagnosis. In the case of changing uptake of MIBG over time, clinical and CT data clearly showed disease progression despite decreasing uptake of MIBG. In none of the four cases was there any delay in the proper diagnosis or staging of the patient's disease.

Our experience with false positives points out other limitations of MIBG scans for liver involvement [4]. Diffuse microabscesses of candidiasis were clinically likely but could not be confidently distinguished from multiple deposits of metastatic disease on MIBG scans. Uptake of MIBG by ganglioneuroma has been reported but is decidedly uncommon [4]. This was one instance where a biopsy was performed to evaluate a persistently abnormal MIBG study due to benign disease.

It has been noted previously that the sensitivity of MIBG for detection of hepatic disease is significantly lower than for detection elsewhere in the abdomen, chest and skeleton [4]. Our experience suggests that MIBG scan alone should not be relied upon for detecting or excluding hepatic neuroblastoma. Once MIBG images are correlated with the clinical picture and the results of higher resolution anatomic imaging studies, few errors and no significant delays in staging or diagnosis should occur. 
Causes of false negative MIBG studies for hepatic involvement which we encountered include inability to distinguish subtle diffuse IV-S disease throughout the liver against normal background uptake, difficulty in resolving small focal lesions, and tumors which do not take up tracer.

\section{References}

1. Shulkin BL, Shapiro B (1990) Radioiodinated metaiodobenzylguanidine (MIBG) in the management of neuroblastoma. In: Po- chedly C (ed) Neuroblastoma tumor biology and therapy. CRC Press, Ann Arbor, pp 171-198

2. Geatti O, Shapiro B, Sisson JC et al (1985) ${ }^{131}$ I-Metaiodobenzylguanidine ( $\left.{ }^{131} \mathrm{I}-\mathrm{MIBG}\right)$ scintigraphy for the localization of neuroblastoma: preliminary experience in 10 cases. J Nucl Med 26: 736

3. Nakajo M, Shapiro B, Copp J et al (1983) The normal and abnormal distribution of the adrenomedullary imaging agent $\mathrm{M}\left[\mathrm{I}^{131}\right]$ iodobenzylguanidine $\left({ }^{131}\right.$-I-MIBG) in man: evaluation by scintigraphy. J Nucl Med 24:672

4. Lumbroso JD, Guermazi F, Hartman O et al (1988) Metaiodobenzylguanidine (MIBG) scans in neuroblastoma: sensitivity and specificity. A review of 115 scans. In: Advances in neuroblastoma research 2, Alan R Liss, pp 689-705

\section{Literature in pediatric radiology}

Journal of Pediatric Surgery (New York)

Late fatal hemorrhage in pediatric liver trauma. Berman, S.S. et al. (Weireter, Jr., L. J., Dept. of Surg., 815 Fairfax Av., Suite 656, Norfolk, VA 23507, USA) 27:1546 (1992)

Inflammatory pseudotumor of the liver in children: report of cases and review of the literature. Hata, Y, et al. (The first Dept. of Surg., Hokkaido Univ. School of Med., N-15 W-7, Sapporo, Japan) 27 1549 (1992)

Isolated hepatic granuloma mimicking congenital simple cyst of the liver possibly caused by tuberculosis. Gündoğdu, Z.H. et al. (Şenocak, M.E. Hacertepe Cocuk Hastanesi, Cocuk Cerrahisi Anabilim Dalt, Ankara 06100, Turkey) 27, 1553 (1992)

An unusual case of choledochal cyst causing hepatic failure requiring orthotopic liver transplantation. Castaldo, P, et al. (Shaw, Jr., B. W., Dept. of Surg., Univ. Med. Center, $600 \mathrm{~S}$ 42nd St., Omaha, NE 68198-3208, USA) 27:1557 (1992)

Pancreatoblastoma resected by delayed primary operation after effective chemotherapy. Inomata, Y. et al. (Dept. of Surg., Central Hosp., 1-1-1 Miwa, Kurashikicity, Okayama 710, Japan) 27: $1570(1992)$

Intrapancreatic duodenal duplication associated with pancreatic pseudocysts. Okuyama, H. et al. (Dept. of Ped. Surg., Univ. Med. School, 1-1-50, Fukushima, Fukushimaku, Osaka 530, Japan) 27: 1573(1992)

Obstructive jaundice associated with polysplenia svndrome in an older child. Stewart, D. E. et al (Cobb, L. M., Jackson Hall, Suite 306, 314 S K St., Tacoma, WA 98405, USA) 27:1575(1992)

Conservative management of splenic abscesses in children. Fernandes, E. T. et al. (Dept. of Urology, Univ. Hosp. and Clinic, 420 Delaware St. SE, Box 394, Minneapolis, MN 55455, USA) 27:1578 (1992)

Primary tubercularabscess of the spleen. Agarwala, S. et al. (Mitra, D.K., Dept. of ped. Surg., All India Inst. of Med. Sciences, New Delhi 110029 India) 27:1580(1992)

Partial situs inversus: duodenal obstruction in a neonate with isolated levocardia. Tryfonas, G.I. et al. (Kath. Rossidi St. 1, GR-54655 Thessaloniki, Greece) 27: 1584 (1992)

Chronic intussusception associated with Yersinia enterocolitica mesenteric adenitis. Hervás, J. A. et al. (Cecilio Metelo 14- $8^{\circ}$ I, Palma de Mallorca, E-07003, Spain) 27: 1591 (1992)
Neuroblastoma masquerading as a retroperitoneal salmonella abscess. Ford, E. G. et al. (Mahour G.H., Head Div. of Ped. Surg., Children's Hosp., 4650 Sunset Blvd., Los Angeles, CA 90027, USA) 27:1608(1992)

Perinatal ovarian cyst: a nonsurgical approach Campbell, B.A. et al. (Garg, R.S., Dept. of Neurol., Med. Univ., Charleston, SC29425, USA) 27 . $1618(1992)$

Prenatal management of congenital cystic adenomatoid malformation of the lung. Dumez, Y. et al. (Port Royal Fetal Med. Unit, 123 Blvd. de Port Royal,F-75014 Paris, France) 28:36 (1993)

Pancreatitis and anomalous union of the pancreaticobiliary ductal system in childhood. Mori, $\mathrm{K}$. et al. (Second Dept. of Surg., School of Med., Univ., 13-1 Takara-machi, Kanazawa 920, Japan) 28:67(1993)

The place of ultrasonographic examination in the initial evaluation of children sustaining blunt abdominal trauma. Akgür, F.M.et al. (Tanyel, F.C., Hacettepe Cocuk Hastanesi, Çocuk Cerrahisi Anabilim Dali, Sihhiye, Ankara 06100, Turkey) 28:78 (1993)

Magnetic resonance imaging of abdominal masses in children. Vade, A., Azienstein, R. (Dept. of Rad., Loyola Univ. Med. Center, 2160 S First Av, Maywood, IL 60153, USA) 28: 82 (1993)

Papillary cystic cancer of the pancreas: diagnostic difficulties. Ward, H.C. et al. (Dept. of Paed. Surg., Inst. of Child Health, Guilford St., London WC1N 1EH, England) 28:89(1993)

Congenital duodenal obstruction: a 32-year review. Bailey,P.V. et al. (Weber, T.R.,Cardinal Glennon Children's Hosp., 1465 S Grand Blvd., St Louis, MO 63104, USA) 28:92(1993)

Mairotation of bowel: variable patterns with different surgical considerations. Schey, W.L. et al (Dept. of Diagn. Rad., Michael Reese Hosp. \& Med. Center, Lakeshore Dr at 31st St., Chicago, IL 60616, USA) 28: 96 (1993)

Posterior mediastinal masses. Saenz, N.C. et al. (Shamberger, R.C., Children's Hosp., Dept. of Surg., Fegan 3, 300 Longwood Av., Boston, MA 02115, USA) 28: 172 (1993)

Rectal manometry, computed tomography, and functional results of anal atresia surgery, Doolin, E. J. et al. (Dept. of Surg., Suite 411, 3 Cooper Plaza, Camden, NJ 08103, USA) 28: 195 (1993)

Megasigmoid: a source of pseudoincontinence in children with repaired anorectal malformations. Peña, A., Behery, M.E. (Ped. Surg., Schneider
Continued from p. 275

Children's Hosp., 269-01 76th Av., New Hyde Park, NY 11042, USA) 28: 199 (1993)

Cecoureterocele: experience of three cases with special reference to the relevance of endoscopic incision. Gotoh, T. etal. (Dept. of Urol. Hokkaido Univ. School of Med., North-15, West-7, Kita-ku, Sapporo060, Japan) 28:223 (1993)

Renal hydatid disease: diagnosis and treatment. Tryfomas, G. J.et al. (Kathigiti Rossidi 1, GR-546 55 Thessaloniki, Greece) 28:228 (1993)

Suprapubic dermoid sinus. Groff, D. B. (Kosair Children's Hosp., P.O. Box 35070, Louisville, KY 40232-5070,USA) 28: 242(1993)

Abdominoscrotal hydrocele in childhood. Wlochynski, T. et al. (Regional Children's Hosp., Dziekanow Lesny, Warsaw, Poland) 28: 248 (1993)

Ingested foreign body presenting as an irreducible inguinal hernia in a baby. Salaman, R, Foster, M (Foster, M., Dept. of Surg., East Glamorgan Hosp., Pontypridd, South Glamorgan, Wales, England) 28: 262 (1993)

\section{Medical and Pediatric Oncology (New York)}

Position paper: imaging methods for primary renal tumors of childhood: costs versus benefits. D'Angio, G. J. et al. (Dept. of Rad. Incol., Hosp. of the Univ., 3400 Spruce St., Philadelphia, PA 19104, USA) 21:205 (1993)

Focal nodular hyperplasia of the liver in childhood. Reymond, D. et al. (D'Angio, G.J., Dept. of Radition Oncol., Hops. of the Univ., 3400 Spruce St., Philadelphia, PA 19104, USA) 21: 283 (1993)

Local control measures in a toddler with a pelvic primitive neuroectodermal tumor. Tendler, C.L. et al. (D'Angio, G. J., Dept. of Radition Oncol., Hops. of the Univ., 3400 Spruce St., Philadelphia, PA 19104, USA) 21:287(1993)

Malignant germ cell tumours in two siblings. Blake, K.I., Gerrard, M.P. (Gerrard, M.P., Dept. of Paed. Oncol.. The Children's Hosp., Western Bank, Sheffield, S102Th, England)21:299(1993)

Comparison of serial PET and MRI scans in a pediatric patient with a brainstem glioma, Bruggers, C.S. et al. (Dept. of Ped., Div. of Hematol./Oncol.. Box 2916, Duke Univ. Med. Center, Durham, NC27710 USA) 21: 301 (1993)

Cerebral non-Hodgkin's lymphoma discovered when treating Hodgkin's disease. Bergeron, C.et al. (Unit. d'Oncol. et Hématol., Péd., Hôpital Sud, 16 Blyd. de Bulgarie, F-35056 Rennes Cedex, France) 21:307(1993) 